\title{
Biopolímeros capaces de reemplazar a los plásticos tradicionales.
}

\section{Biopolymers capable of replacing traditional plastics}

Natalia Sofía Barahona Alvear. ${ }^{1}$, Hernán Patricio Tixi Toapanta. ${ }^{2}$, Segundo Hugo Calderón. ${ }^{3}$ \& Lorena Barahona Alvear. ${ }^{4}$

Recibido: 01-08-2019 / Revisado: 25-08-209 /Aceptado: 04-09-2019/ Publicado: 04-10-2019

\begin{abstract}
.
DOI: https://doi.org/10.33262/cienciadigital.v3i4.954

The production of bioplastics, with the use of raw materials such as carbohydrates (starch), protein (gelatin) and with the help of a plasticizer (glycerol) can replace synthetic plastics that are not biodegradable and are the great pollutants of the environment. In this investigation, bioplastics were prepared in combination of these raw materials in various proportions and concentrations together with the plasticizer that is diluted in water. Which were subjected to mechanical tests such as; Tensile strength, percentage of final elongation, modulus and hardness, a device built for this purpose was used that represents a good tool for the analysis and calculation of these tests. Results were obtained in which it is indicated that the properties of the bioplastics obtained have mechanical properties similar to low density polyethylene and high impact polystyrene that can be used for packaging material, whether in the manufacture of items such as toys, utensils Disposable table tops, it is also important to indicate that these bioplastics can be subjected to other biopolymer refining procedures and be used in pharmaceutical medicaments for coating tablets. The main objective of this project was to carry out an experimental analysis with different concentrations of solvent and plasticizer for the production of bioplastic from carbohydrates (starch) and protein (gelatin), which was developed over a period of 2 years.
\end{abstract}

\footnotetext{
1 Escuela Superior Politécnica de Chimborazo/ Facultad de Mecánica/ Escuela de Mantenimiento Industrial, natalia.barahona@espoch.edu.ec

${ }^{2}$ Escuela Superior Politécnica de Chimborazo/ Facultad de Ciencias/ Escuela de Ingeniería Ambiental, htixi@espoch.edu.ec

3 Escuela Superior Politécnica de Chimborazo/ Facultad de Ciencias/ Escuela de Ingeniería Química, hcaldero@espoch.edu.ec

${ }^{4}$ Ginfar, Chimborazo, Ecuador, loro.barahona @ hotmail.com
} 
Keywords: Bioplastics, starch, gelatin, glycerol

\section{Resumen.}

La elaboración de bioplásticos, con la utilización de materias primas como carbohidratos (almidón), proteína (gelatina) y con la ayuda de un plastificante (glicerol) pueden sustituir a los plásticos sintéticos que no son biodegradables y son los grandes contaminantes del medio ambiente. En esta investigación se realizó la elaboración de bioplásticos en combinación de estas materias prima en diversas proporciones y concentraciones junto con el plastificante que es diluido en agua. los cuales fueron sometidos a pruebas mecánicas como son; resistencia a la tensión, porcentaje de elongación final, módulo y dureza, se utilizó un equipo construido para este propósito que representa una buena herramienta para el análisis y cálculo de dichas pruebas. Se obtuvieron resultados en la que se indica que las propiedades de los bioplásticos obtenidos tienen propiedades mecánicas parecidas al polietileno de baja densidad y al poliestireno de alto impacto que pueden ser utilizados para material de empaque, ya sea en la fabricación de artículos como juguetes, utensilios de mesa desechables respectivamente, es también importante indicar que estos bioplásticos pueden ser sometidos a otros procedimientos de refinamiento del biopolímero y ser utilizados en medicamentos farmacéuticos para recubrimiento de tabletas.

Este proyecto tuvo como objetivo principal realizar un análisis experimental con distintas concentraciones de disolvente y plastificante para la producción de bioplástico a partir de carbohidratos (almidón) y proteína (gelatina) el cual se desarrolló en un tiempo de 2 años.

Palabras claves: Bioplásticos, almidón, gelatina, glicerol

\section{Introducción.}

El plástico es parte de nuestro diario vivir, actualmente su evolución se ha estudiado durante años debido a los beneficios que posee y su accesibilidad ha logrado que hasta hoy se analice y se mejore cada vez más su composición, en adición, ha traído muchos avances y desarrollo a la humanidad junto con la tecnología, pero su producción elevada en el mundo sobre todo en el medio ambiente ha producido y produce efectos adversos, lo que ha generado fuertes necesidades de encontrar sustitutos óptimos a las características que brindan los plásticos convencionales para sus distintos usos.(1)

El plástico sintético es fabricados a partir de derivados del petróleo como el polietileno, polipropileno, policloruro de vinilo y el polietilen tereftalato, los cuales no son biodegradables y representan un serio problema de contaminación ambiental, siendo los causantes de problemas de salud al consumir alimentos y bebidas en este tipo de envases. (2). Los bioplásticos son una alternativa para disminuir la contaminación producida por los plásticos sintéticos, ya que son 
polímeros que provienen de fuentes naturales y renovables, todos son biodegradables por microorganismos como bacterias, hongos y algas. (3). La degradación de los bioplásticos es el resultado de la producción de dióxido de carbono, agua y humus. (3). Los carbohidratos y proteínas, son la materia prima para obtener los bioplásticos por eso son considerados plásticos biodegradables y materiales considerados como un recurso renovable que tienen su origen en materias orgánicas. (4).

Los primeros bioplásticos vegetales, fueron fabricados a partir de almidón de patata, maíz o yuca. Sus polímeros de glucosa sirven para que, tras un proceso de extrusión y otros tratamientos acaben convirtiéndose en material para cubiertos, envases o bolsas, los bioplásticos tienen, aplicaciones prometedoras en la protección del medio ambiente que podrían llegar a sustituir a los plásticos de uso tradicional (5). En estudios realizados la producción del plástico biodegradable empieza con el almidón que se extrae del maíz, luego los microorganismos lo transforman en una molécula más pequeña de ácido láctico que sirve como base para la elaboración de cadenas poliméricas de ácido poliláctico.(6), transformándose en productos biodegradable o sustancia que puede descomponerse en los elementos químicos que lo conforman, debido a la acción de agentes biológicos, como plantas, animales, microorganismos y hongos, bajo condiciones ambientales naturales que pueden contribuir de manera efectiva tanto a la protección del ambiente como al desarrollo de la industria del plástico en sí. Sin embargo, es necesario conocer sus propiedades y características, de forma que las aplicaciones a las que se destinen se beneficien de las mismas. (6)

En un estudio reciente publicado en la revista "Science" señala que hay plástico presente en los mares de todo el mundo (7). por este motivo es necesario el reemplazo de los plásticos no degradables por biopolímeros totalmente degradables obtenidos a partir de fuentes de orgánicas renovables para una solución en gran medida más completa para los diferentes aspectos de este problema. Sin embargo, el precio de los bioplásticos sigue siendo demasiado alto como para que puedan desplazar a los plásticos tradicionales. Debido a esto, es necesario diseñar estrategias para obtener bioplásticos a un bajo costo o similar al plástico convencional. Es importante también señalar que la biodegradabilidad de los bioplásticos es una propiedad que depende de la estructura química del material, es independiente del origen o fuente de obtención del bioplásticos que pueden ser fuentes no renovables que son biodegradables, así como bioplásticos procedentes de fuentes renovables que no lo son y sus propiedades varían dependiendo del material del que se trate (8).

Otro aspecto importante mencionar es el mercado de los envases fabricados con bioplásticos ya que es un mercado alternativo o de sustitución, y pretende sustituir un porcentaje de envases convencionales por envases biodegradables. (8). En este mercado, la oferta depende básicamente del precio del petróleo, que determina el precio de los envases fabricados con polímeros no biodegradables y marca, por tanto, la competitividad y productividad depende de esto. La demanda de los bioplásticos se debe generar desde las empresas productoras, apelando a un sentido más ecológico y de consumo responsable, a través del empleo de envases biodegradables. Para que 
exista esta demanda se debe lograr la biodegradabilidad total del envase. conseguir una respuesta positiva de los consumidores al precio de los envases biodegradables, que hoy en día son de 2 a 4 veces más altos que los de los envases convencionales, fabricar bioplásticos a partir de cultivos que no sean utilizados para la alimentación humana (9). Los sectores más importantes a los que van destinados estos polímeros biodegradables son: envases y bolsas de la compra en comercio, maquinillas de afeitar y otros enseres, eléctrico-electrónico (ordenadores, fotografía), automóvil (revestimientos interiores y salpicaderos), sanitario (prótesis), agrícola (plásticos para invernaderos). (9)

La viabilidad de la utilización de los bioplásticos en envases depende de las características intrínsecas del plástico fabricado y del uso que se le vaya a dar, estas pueden ser: barrera al aire (oxígeno), barrera frente a la humedad o vapor de agua, propiedades térmicas, propiedades mecánicas, migraciones, contaminación microbiana, resistencia a la penetración de microorganismos en los envases, resistencia al agua (impermeabilización). En el sector de envases y embalajes, la tendencia irá dirigida, posiblemente, a incorporar en el mercado bolsas de la compra biodegradables y plástico compostable para la agricultura. En cuanto a envases para alimentos, la tendencia se dirige hacia el empleo del PLA y PHB, una vez alcanzados los resultados tecnológicos óptimos requeridos para el envasado alimentario mediante la adición de nanomateriales, actualmente en fase de investigación. El alcanzar las capacidades productivas anteriormente comentadas, así como los porcentajes de sustitución, implica destinar la superficie de cultivo de las materias primas de las que se obtienen los bioplásticos. (10)

Dicho esto, los autores de esta investigación vieron la necesidad de hacer estudios experimentales sobre el proceso de producción de bioplásticos por la gran contaminación ambiental que vivimos expuestos y de esta manera contribuir de alguna manera a su no contaminación ambiental. Esto llevó a cuestionarse si se puede o no obtener bioplásticos a partir de materias primas que se encuentran a nuestro al canse y poder sustituir al plástico tradicional.

Este trabajo busca brindar a la sociedad una nueva alternativa para solucionar los graves problemas ambientales debido a la contaminación con desechos plásticos que en la actualidad constituyen uno de los principales dolores de cabeza, es necesarios recurrir aquellos materiales que son de origen natural como son las proteínas y carbohidratos que son biodegradables y sus elementos constitutivos regresaran al medio ambiente.

El objetivo general del trabajo aquí expuesto consiste en la elaboración de biopolímeros utilizando combinaciones diferentes de gelatina, almidón, y como agente plastificante el glicerol, teniendo materiales muy parecidos a filmes plásticos convencionales, con el fin de evaluar pruebas mecánicas: fuerzas de tensión comprensión e impacto, que se analizaron mediante un aparato simple, barato, y absolutamente instructivo diseñado para medir características de los biopolímeros, por su enorme efecto sobre nuestra vida diaria. 
El presente artículo consta de: materiales y métodos, resultados con su respectivo análisis y por último conclusiones.

\section{Metodología}

El trabajo experimental tuvo una duración de dos años, durante los cuales se obtuvieron los biopolímeros para la determinación de sus propiedades mecánicas como son Resistencia, Porcentaje de Elongación, y Dureza, estas pruebas se determinaron mediante el equipo que se construyó para este propósito, utilizando como materia prima carbohidratos (maicena), proteína (gelatina) y como plastificante el glicerol con el que se elaboraron los biopolímeros. La Figura uno muestra un dispositivo sencillo para estimar las propiedades mecánicas que puede ser construido de manera relativamente fácil.

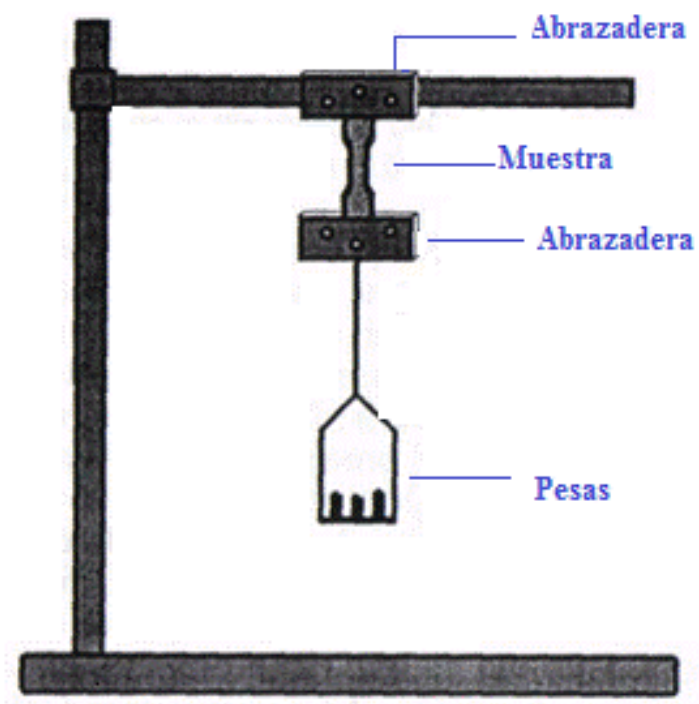

Figura 1: Equipo para medir propiedades Mecánicas

Fuente: Elaboración Propia

\section{Materiales}

Materia Prima: Almidón (maicena IRIS) disponible en tiendas y supermercados, gelatina sin sabor, de la marca ROYAL disponible en tiendas y supermercados

Insumos: Glicerol líquido (1\%), incoloro y de viscosidad media (como plastificante); agua destilada; colorante vegetales (verde, rojo, amarillo, azul), base ftalocianina de carácter orgánico, material de teflón

Equipo: Se lo construyó desde una posición de anillo de laboratorio sujetada en la vara de apoyo horizontal, siendo el apoyo una balanza primaveral. Las dos abrazaderas de la muestra pueden ajustarse fácilmente por la acción de cuatro agujeros taladrados los agujeros se pueden atar a la vara de apoyo de encima, y a la balanza piramidal. Dos agujeros adicionales en cada par, junto con cuatro tornillos pequeños y nuez, permite apretar las abrazaderas después de que una muestra se 
inserta. Una muestra se sujeta en el lugar, y se agregan los pesos en la balanza. Los pesos proporcionan una tensión creciente. La muestra se pondrá tensa, y puede alargarse hasta cierto punto y se romperá. El resultado de este procedimiento simple se llamaría la fuerza tensora. Los pesos agregados proporcionarán una indicación de la fuerza tensor, si los pesos se agregan despacio, con los intervalos de tiempo cortos, y el mismo procedimiento se usa para cada prueba sucesiva. Cómo se puede repetir el procedimiento pueden ser probados repitiendo la medida con dos o más muestras idénticamente preparadas.

\section{Métodos}

El método utilizado es experimental, elaboración de bioplásticos (método de Films).

Las pruebas se realizan utilizando el equipo mencionado anteriormente con cuatro probetas para cada bioplásticos elaborado, para determinar la resistencia tensil, elongación, módulo y dureza el cual es comparado con el valor de la resistencia del LDPE.

Método de Filmes (elaboración de bioplásticos): Este método se basa en la obtención de biopolímeros o películas (film) mediante la preparación de solución y luego la evaporación del solvente agua. Se utilizó gelatina sin sabor, almidón (maicena) y glicerol como plastificante y el agua que actúa como un solvente. Los componentes se seleccionaron en base a su costo bajo y disponibilidad.

Obtención del biopolímero: Se realizó combinaciones de gelatina sin sabor, almidón y glicerol. En la tabla uno y dos se puede apreciar las combinaciones de gelatina y almidón para la obtención del biopolímero, se mezclaron los ingredientes sin importar el orden, a una temperatura de $85^{\circ} \mathrm{C}$ eliminando la posible formación de pequeñas burbujas ya que pueden producir agujeros en los filmes y llevar a moldes de teflón iniciando el proceso de secado del film que dura aproximadamente un día.

Preparación de la muestra: Se cortó la muestra en forma perro-hueso usando un estilete. En la figura dos se puede apreciar la forma en la que se tiene que cortar cada una de las muestras de film, la muestra tuene que tener un ancho 0.25 pulgada ( 0.64 centímetro).

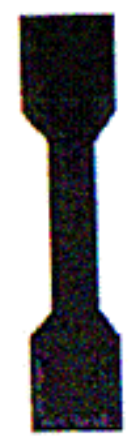

Figura 2: Plantilla perro-hueso

Fuente: Elaboración propia 
A continuación se presenta la tabla uno y dos con las combinaciones de gelatina y almidón para las muestras analizadas.

Tabla 1: Combinaciones de gelatina-glicerol-agua

\begin{tabular}{cccc}
\hline $\mathbf{N}^{\mathbf{0}}$ Muestra & gelatina(g) glicerol(mL)agua $(\mathbf{m L})$ \\
\hline $1 \mathrm{~A}$ & 2.25 & 60 & 30 \\
$2 \mathrm{~A}$ & 2.25 & 30 & 30 \\
$3 \mathrm{~A}$ & 2.25 & 60 & 60 \\
$4 \mathrm{~A}$ & 2.25 & 90 & 30 \\
$5 \mathrm{~A}$ & 3.75 & 60 & 30 \\
$6 \mathrm{~A}$ & 3.75 & 90 & 90 \\
$7 \mathrm{~A}$ & 3.75 & 60 & 60 \\
$8 \mathrm{~A}$ & 3.75 & 90 & 30 \\
$9 \mathrm{~A}$ & 5 & 90 & 45 \\
$10 \mathrm{~A}$ & 5 & 60 & 45 \\
$11 \mathrm{~A}$ & 5 & 60 & 30 \\
$12 \mathrm{~A}$ & 7.5 & 120 & 60 \\
$13 \mathrm{~A}$ & 7.5 & 120 & - \\
$14 \mathrm{~A}$ & 7.5 & 180 & 90 \\
\hline
\end{tabular}

Tabla 2: Combinaciones de almidón-glicerol-agua

$\overline{N^{0}}$ Muestraalmidón $(\mathrm{g})$ glicerol $(\mathrm{mL})$ agua $(\mathrm{mL})$

\begin{tabular}{cccc}
\hline 1B & 1.5 & 120 & - \\
$2 \mathrm{~B}$ & 2.5 & 60 & 30 \\
\hline
\end{tabular}




\begin{tabular}{cccc}
\hline 3B & 2.5 & 30 & 30 \\
4B & 2.5 & 90 & 30 \\
5B & 3.0 & 60 & 30 \\
6B & 3.0 & 90 & 30 \\
$7 \mathrm{~B}$ & 3.0 & 90 & 45 \\
8B & 4.5 & 90 & 30 \\
9B & 4.5 & 90 & 45 \\
$10 \mathrm{~B}$ & 4.5 & 180 & 90 \\
11B & 4.5 & 180 & 30 \\
12B & 7.0 & 90 & 45 \\
13B & 7.0 & 120 & - \\
14B & 7.0 & 180 & 90 \\
\hline
\end{tabular}

\section{Pruebas Mecánicas}

\section{Resistencia a la Tensión}

Método: Determinación de resistencia a la tensión en el punto de rompimiento del biofilm. Se cortan cuatro probetas de 0,25 pulgadas esta dimensión corresponde a la sección más angosta del film, los espesores de cada probeta fueron medidas utilizando un micrómetro de tornillo, se colocó en las mordazas del equipo superior e inferior, hay que tener cuidado de no ajustar mucho para que no se rompa la muestra, se coloca las pesas en la balanza del equipo, se anota pesos totales en los cuales se produjo el rompimiento de los cuatro ensayos. Cabe recalcar que los análisis son válidos solamente cuando la probeta sufre el rompimiento en su sección más angosta. Los valores reportados corresponden al promedio de cuatro mediciones en cada tratamiento.

\section{Porcentaje de Elongación:}

Método: Porcentaje de elongación final en el momento de la ruptura. De igual manera se procede como en el caso anterior de la resistencia, se utilizan cuatro lecturas para cada film y los valores reportados corresponden al promedio de cuatro mediciones en cada tratamiento. Este procedimiento se lo realiza simultáneamente con la determinación de la resistencia y se basa en cuantificar la elongación total de la probeta hasta el momento de su rompimiento. 


\section{Módulo:}

Método: Determinación de módulo tensil en el punto de rompimiento del film Para medir el módulo ténsil, hacemos lo mismo que para medir la resistencia y la elongación final.

\section{Dureza:}

Método: Permite conocer el grado de dureza del material. Se siguen los mismos pasos anteriores, el film que tenga mayor peso será el más resistente a ser quebrado.

Diseño Experimental: Se aplicó análisis de varianza con un nivel de confianza del 95\% a un factor para las muestras de bioplásticos obtenidas experimentalmente cada uno con cuatro réplicas de catorce muestras preparadas, utilizando gelatina-glicerol-agua y almidón-glicerol-agua para determinar si es que existen combinaciones que proporcionen un nivel de resistencia significativamente mayor que las demás y posteriormente se aplica t-studen para la comparación de medidas.

\section{Resultados}

\section{Resistencia a la Tensión}

La resistencia a la tensión para la muestra 14A, con un análisis de varianza de un solo factor de $3.35 \mathrm{E}+28$ tiene diferencias significativas $(\alpha<0.05)$ con respecto a las trece muestras analizadas, es decir tiene mejor resistencia a la tensión que el resto, con respecto a las muestras de almidónglicerol-agua las muestras 12B y 13B; con un análisis de varianza de $2.71 \mathrm{E}+22$ y de $7.00 \mathrm{E}+20$ tienen una resistencia a la tensión mejor que el resto de muestras analizadas.

\section{Porcentaje de elongación final (\% Ef.)}

El porcentaje de elongación final indica, que la muestra antes de romperse sufre un estiramiento de tal manera que se deforma. Para bioplásticos de gelatina-glicerol-agua en el análisis de varianza de un solo factor indica que cuatro de las muestras analizadas presentan mejores resultados a la deformación, es decir no todas las muestras antes de romperse tienen porcentaje de elongación similar. Los bioplásticos con mejor \% Ef. son 6A, 12A, 13A y 14A. De la misma forma al ser sometidos los bioplásticos de almidón-glicerol-agua a este análisis de varianza de un solo factor se puede ver que las muestras 5B, 7B, 8B y 14B tienen mejor \% Ef. que el resto de muestras. En la tabla tres y cuatro se resume el cálculo de análisis de varianza a un factor para las muestras de bioplásticos preparadas de gelatina-glicerol-agua y almidón-glicerol-agua, para ver si existe alguna combinación que proporciona un porcentaje de elongación final significativamente mayor que las demás. 
Tabla 3: \%Ef. gelatina-glicerol-agua análisis de varianza de un solo factor

\begin{tabular}{ccccc}
\hline Análisis de varianza de un factor & \multicolumn{4}{c}{ \%Ef. GELATINA- GLICEROL- AGUA } \\
\hline RESUMEN & & & & \\
Muestras & N $^{\text {repetición }}$ muestras & Suma & Promedio Varianza \\
1 A & 4 & 400,23 & 100,06 & $3,96 \mathrm{E}-03$ \\
2A & 4 & 413,83 & 103,46 & $8,79 \mathrm{E}-01$ \\
3 A & 4 & 417,35 & 104,34 & $1,65 \mathrm{E}+00$ \\
4 A & 4 & 406,17 & 101,54 & $1,39 \mathrm{E}-01$ \\
5 A & 4 & 413,06 & 103,27 & $1,10 \mathrm{E}-01$ \\
6 A & 4 & 416,73 & 104,18 & $1,59 \mathrm{E}-02$ \\
7 A & 4 & 406,25 & 101,56 & $1,63 \mathrm{E}-03$ \\
8 A & 4 & 403,93 & 100,98 & $2,34 \mathrm{E}-02$ \\
9 A & 4 & 405,88 & 101,47 & $6,87 \mathrm{E}-03$ \\
10 A & 4 & 411,52 & 102,88 & $3,13 \mathrm{E}-02$ \\
11 A & 4 & 402,91 & 100,73 & $1,06 \mathrm{E}-02$ \\
12 A & 4 & 421,54 & 105,39 & $4,30 \mathrm{E}-03$ \\
13 A & 4 & 422,03 & 105,51 & $9,14 \mathrm{E}-02$ \\
14 A & 4 & 423,5 & 105,88 & $1,67 \mathrm{E}-04$
\end{tabular}

Tabla 4: \%Ef. almidón-glicerol-agua, análisis de varianza de un solo factor

\% Ef. ALMIDON-GLICEROL-AGUA

RESUMEN 


$\begin{array}{ccccc}\text { Grupos } & N^{0} \text { repetición muestras Suma Promedio Varianza } \\ \text { 1B } & 4 & 441,37 & 110,34 & 4,56 \mathrm{E}-01 \\ \text { 2B } & 4 & 456,03 & 114,01 & 5,94 \mathrm{E}-01 \\ \text { 3B } & 4 & 453,38 & 113,35 & 1,67 \mathrm{E}-04 \\ \text { 4B } & 4 & 426,74 & 106,69 & 1,67 \mathrm{E}-04 \\ \text { 5B } & 4 & 486,74 & 121,69 & 4,32 \mathrm{E}-01 \\ \text { 6B } & 4 & 441,34 & 110,34 & 4,49 \mathrm{E}-01 \\ \text { 7B } & 4 & 494,88 & 123,72 & 5,02 \mathrm{E}-01 \\ \text { 8B } & 4 & 493,85 & 123,46 & 8,69 \mathrm{E}-01 \\ \text { 9B } & 4 & 454,72 & 113,68 & 4,36 \mathrm{E}-01 \\ \text { 10B } & 4 & 416,44 & 104,11 & 6,87 \mathrm{E}-03 \\ \text { 11B } & 4 & 435,19 & 108,80 & 4,36 \mathrm{E}-01 \\ \text { 12B } & 4 & 408,05 & 102,01 & 5,86 \mathrm{E}-01 \\ \text { 13B } & 4 & 414,05 & 103,51 & 2,12 \mathrm{E}+00 \\ \text { 14B } & 4 & 500,27 & 125,07 & 4,06 \mathrm{E}+00\end{array}$

Al aplicar el t-student para la comparación de medidas entre los bioplásticos de gelatina-glicerolagua con las de almidón-glicerol-agua, sugiere que la preparación con almidón posee un porcentaje de elongación significativamente mayor que la preparación con gelatina. En la tabla cinco se indican las muestras de almidón y gelatina con mejores porcentajes de elongación.

Tabla 5: Comparación de \%Ef. entre muestras de almidón y gelatina

$\overline{\mathbf{N}^{0}}$ Muestra \%Ef. Almidón \%Ef. Gelatina No Muestra

\begin{tabular}{cccc}
\hline 6 & 121.69 & 104.18 & 5 \\
12 & 123.72 & 105.39 & 7 \\
13 & 123.46 & 105.51 & 8 \\
14 & 125.07 & 105.88 & 14 \\
\hline
\end{tabular}


En la figura tres se puede apreciar que existe diferencia significativa entre las composiciones de gelatina y de almidón, presentando variación en sus datos, indicando que las muestras de almidón tienen un mejor porcentaje de elongación.

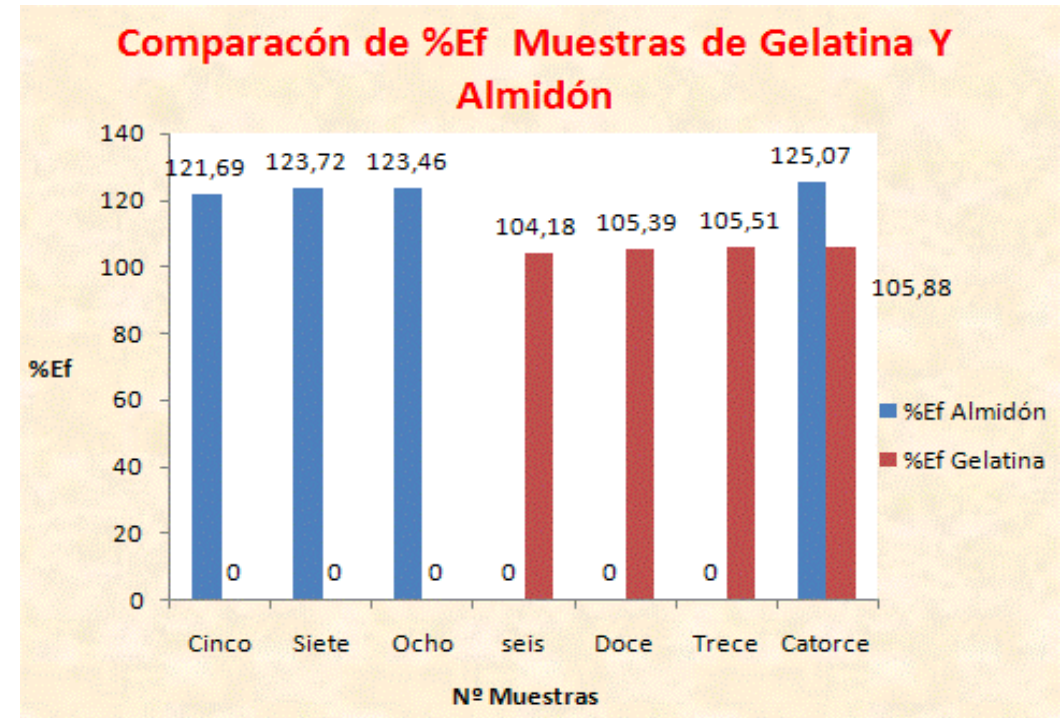

Figura 3: Comparación \%Ef. entre muestras de gelatina y almidón.

Fuente: Elaboración propia

Al compararse entre sí las muestras de almidón se puede apreciar que la muestra catorce tiene mejor porcentaje de elongación a diferencia de las otras esto se puede evidenciar en la figura cuatro

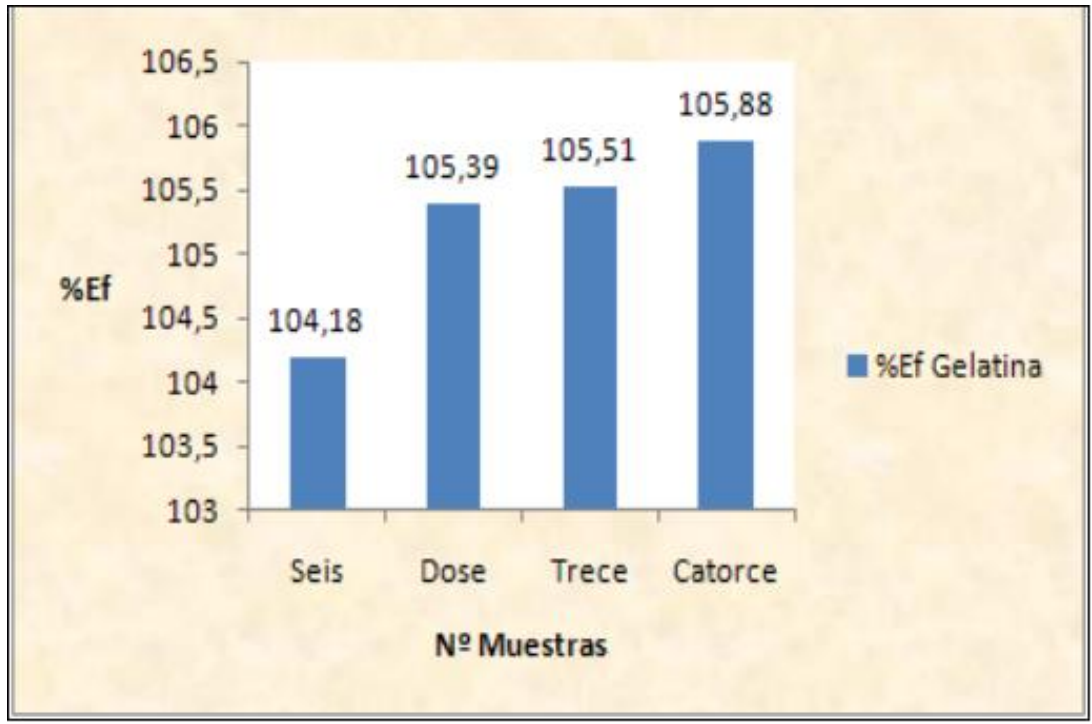

Figura 4: Comparación \%Ef. entre biopolímeros de gelatina.

Fuente: Elaboración propia 
Gráficamente podemos apreciar en la figura 5 que los datos presentan variación entre muestras de almidón, es decir no todas la muestras tienen porcentaje de elongación final similares o equivalentes ya que el $t$ calculado es mayor que el $t$ crítico y que a su vez la muestra 14B es el que presenta mejor `porcentaje de elongación a diferencia de las demás muestras analizadas

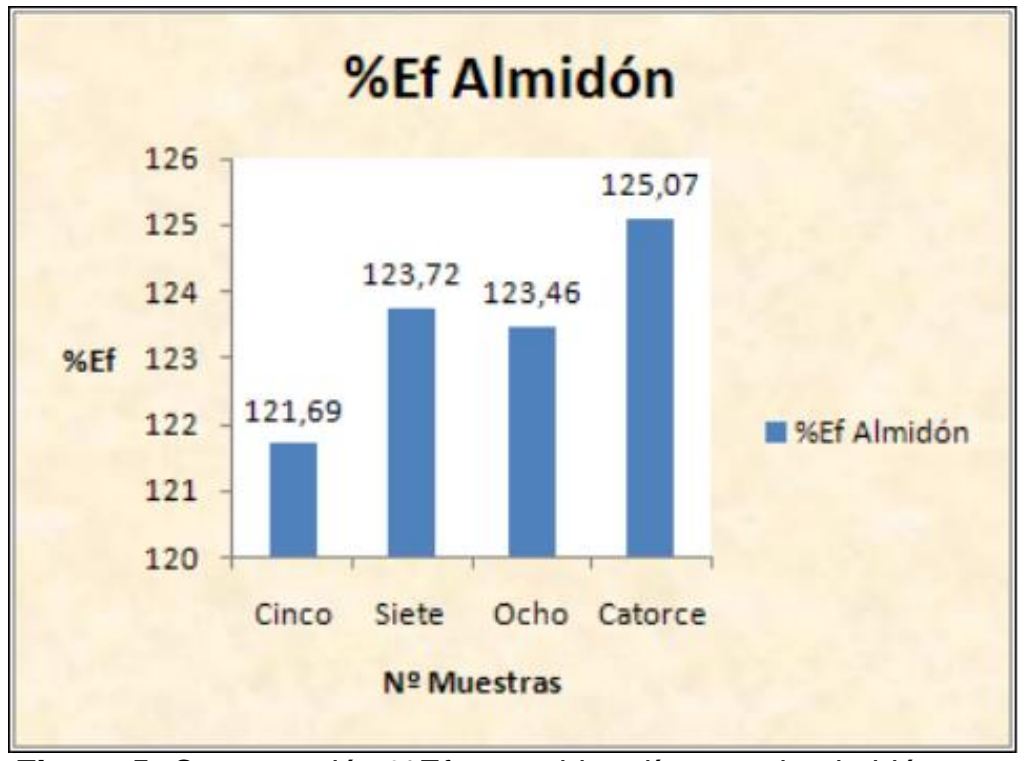

Figura 5: Comparación \%Ef. entre biopolímeros de almidón.

Fuente: Elaboración propia

\section{Módulo}

Según el cálculo del e varianza a un factor para muestras de gelatina-glicerol-agua, la muestra 14A tiene el mejor modulo $(7,13 \mathrm{E}+23)$ a diferencia de los demás biopolímeros siendo el responsable del valor positivo en el t-student. En los biopolímeros de almidón se encontró que las muestras $12 \mathrm{~B}$ y $13 \mathrm{~B}$ tienen los módulos más grandes $2,20 \mathrm{E}+18$ y $1,67 \mathrm{E}+16$ respectivamente y son significativamente mejor siendo los responsables del resultado positivo del test. Se hace la representación gráfica de la tensión versus elongación, de muestras de gelatina 13A y 14A (mejor porcentaje de elongación y de resistencia) como se indica en la figura seis. La pendiente representa el módulo, la muestra 13A tiene menor pendiente y posee bajo módulo tensil que la muestra 14A tiene una pendiente pronuncias con un alto módulo tensil, lo que significa que es resistente a la deformación, siendo esta más resistente que la anterior. 


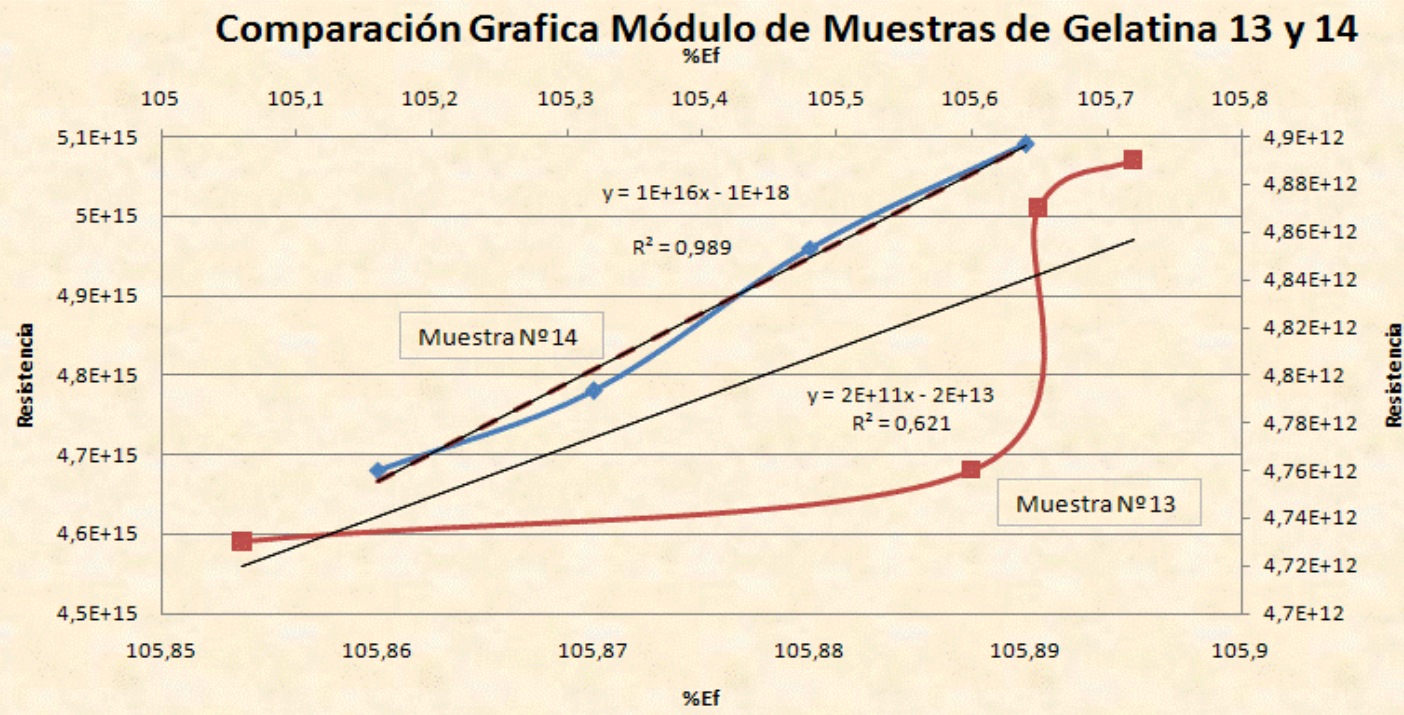

Figura 6: Curva tensión estiramiento (módulo) para bioplásticos de gelatina-glicerol-agua 13A y 14a

Fuente: Elaboración propia

De igual manera se hace la comparación de los mejores bioplásticos de almidón con mejor porcentaje de elongación y de resistencia entre las muestras 12B y 13B como se indica en la figura siete. La muestra 12B tiene una pendiente pronunciada, con un alto módulo ténsil, lo cual significa que es resistente a la deformación. La muestra 13B tiene una pendiente más suave, y posee bajo módulo ténsil en relación a la muestra 12B, pero alta en relación con las demás muestras de almidón y puede ser deformada más fácilmente que la muestra 12B

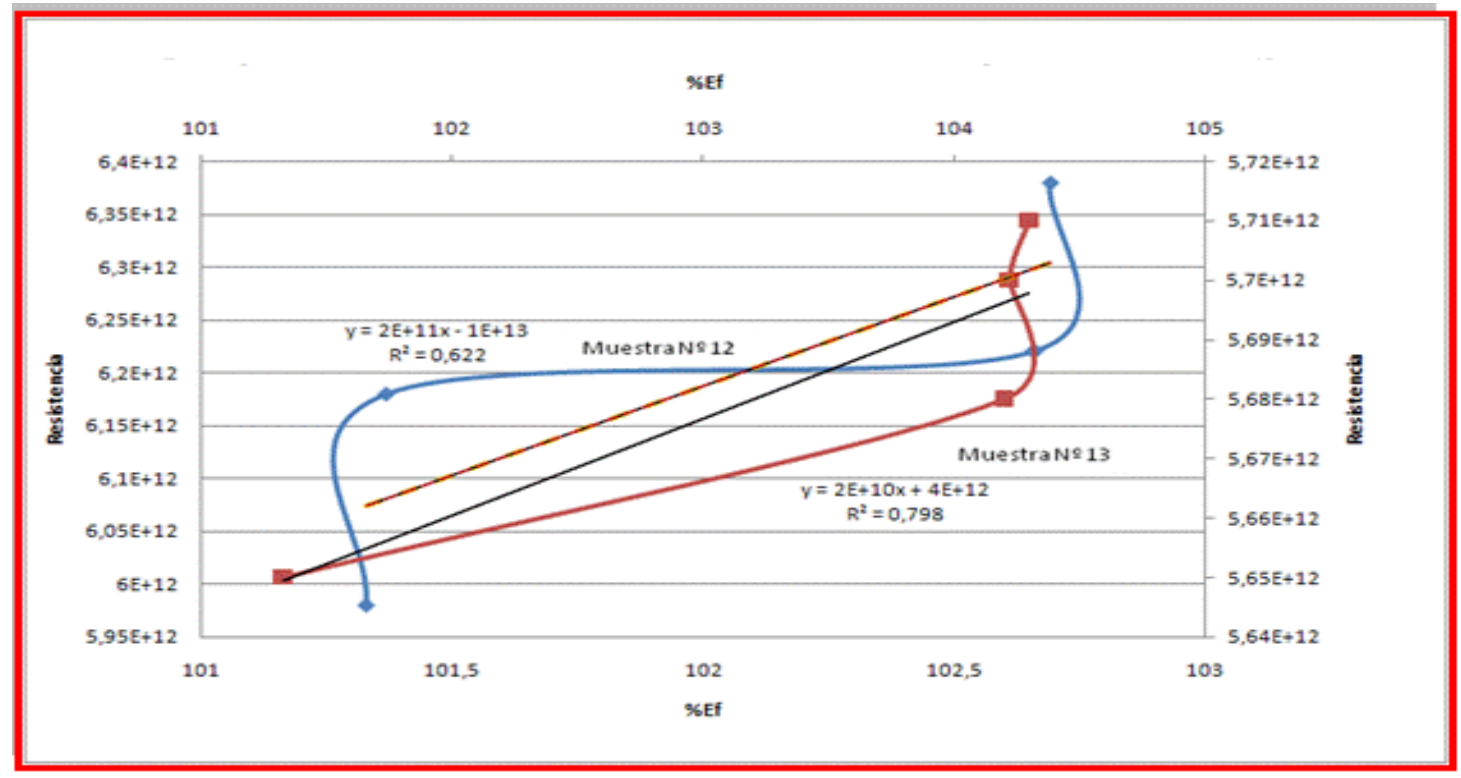

Figura 7: Curva tensión estiramiento (módulo) para bioplásticos de almidón-glicerol-agua 12B y 13B Fuente: Elaboración propia 


\section{Dureza}

Para el cálculo de la dureza que es una medida de energía que una muestra puede absorber antes de romperse, para lo cual trabajamos con el área bajo la curva aplicando una determina fuerza sobre un objeto y eso es igual a la energía que absorberá la muestra antes de romperse. Nótese que el trabajo es igual al área bajo la curva, entonces va ser igual a un medio de la pendiente por el área de la probeta al cuadrado. Las muestras $14 \mathrm{~A}$ y $12 \mathrm{~B}$ presentan una dureza de $2.50 * 10^{15} \mathrm{~J}$ y $1.56 * 10^{10} \mathrm{~J}$ respectivamente. Es decir, la muestra $14 \mathrm{~A}$ absorbe más energía antes de romperse que la muestra $12 \mathrm{~B}$.

Todos estos datos al ser comparados por un estudio realizado por Diana Paola Navia-Porras y Nathalia Bejarano-Arana en la Evaluación de propiedades físicas de bioplásticos obtenidos a partir de almidón de yuca dicen que el comportamiento en las propiedades mecánicas aplicadas a muestras de bioplásticos a partir de almidón de yuca en su análisis de varianza establecen que cada una de las pruebas mecánicas aplicadas varían significativamente $(\mathrm{p}<0,05)$, lo que indica que los bioplásticos obtenidos en este estudio a base de gelatina y almidón de maíz están dentro de los rangos establecidos ya que el comportamiento mecánico $(\sigma \mathrm{F}, \sigma \mathrm{T}, \mathrm{y} \mathrm{ET})$ de los materiales de este estudio fue superior a los bioplásticos obtenidos por Navia en 2011. Este comportamiento puede ser atribuido al uso de proteína como es la gelatina la cual está potenciando su resistencia ante los esfuerzos mecánicos ejercidos favoreciendo sus propiedades funcionales.

En otros estudios, se han reportado valores en propiedades mecánicas similares a los encontrados en esta investigación, tal es el caso de bioplásticos basados en almidón de yuca con alcohol polivinílico $(\mathrm{PVOH})$ y aceites esenciales elaborados por termo-compresión; materiales espumados obtenidos por extrusión de almidón de papa reforzados con fibra de coco cuyo sT <1 MPa (10), y bioplásticos.

Por otra parte, al usar glicerol como plastificante, se confiere a las películas mayor afinidad por las moléculas de agua debido a su carácter hidrofílico. Algunos autores (9.10) usaron glicerol y sorbitol como plastificantes en diferentes proporciones y encontraron que las propiedades mecánicas se ven afectadas por el tipo y cantidad de plastificante ya que, al aumentar el porcentaje de glicerol, el esfuerzo a la tensión disminuyó.

\section{Conclusiones.}

- Las pruebas mecánicas a las cuales fueron sometidos los bioplásticos con un equipo simple, barato, y absolutamente instructivo que fue diseñado para medir características mecánicas que puede ser utilizado a nivel de laboratorio sin la necesidad de recurrir a equipos caros, permitió observar que los valores obtenidos para la muestra 14A presentaron diferencias significativas respecto a las demás muestra analizadas tanto de gelatina como de almidón. Las pruebas mecánicas para los bioplásticos de almidón indican que el esfuerzo y la elongación combinados con su baja resistencia y su baja opacidad hacen que sea un material muy poco atractivo para sustituir algún plástico convencional. Por otro lado, la 
mayor resistencia presentada por los filmes de gelatina combinados con su aceptable transparencia y textura los constituye como elementos muy atractivos para poder sustituir a un plástico tradicional. La muestra 13A podría reemplazar al polietileno de baja densidad como material de empaque, la muestra 14A podrían reemplazar al poliestireno de alto impacto en la fabricación de artículos como juguetes, utensilios de mesa desechables, empaques y luego de someterlos a otros procedimientos de refinamiento del biopolímero se sugiere también utilizar para medicamentos farmacéuticos específicamente para recubrimiento de tabletas. El resto de formulaciones de gelatina se recomiendan ser utilizadas para la elaboración de fundas plásticas biodegradables contribuyendo de esta manera a una menor contaminación ambiental.

\section{Referencias bibliográficas.}

Andes. (febrero de 2016). Obtenido de http://www.andes.info.ec/es/noticias/ecuador-identifica2014-ano-ciencia-tecnologia-educacion-superior.html

Almeida, A., Ruiz, J., López, N., \& Pettinari, J. (2916). Bioplásticos: una alternativa ecológica. Revista QuímicaViva número 3, 10-13.

Angeles, A. (2016). "Obtención de biopolímeros plástico a partir de almidón de malanga. Scielo, $2-4$

Cortés, J., Fernández, A., Mosquera, S., \& Velasco, R. (2017). Evaluación de propiedades mecánicas, ópticas y de barrera en películas actuvas de almid;on de yuca. Scielo, 1-5.

García Torres, \& Pulido Bonilla. (2018). Bioplásticos fotoactivos. Alternativa frente a procesos oxidativos en alimentos de. XX Congreso Internacional Inocuidad de los alimentos, 2-3. Obtenido de Bioplásticos fotoactivos. Alternativa frente a procesos oxidativos en alimentos de: file:///C:/Users/HP2540P/Downloads/344-1732-1-PB.pdf

Luna, G. (2917). Almidón termoplástico de yuca reforzado con fibra de fique: preliminares. Revista Dyna, 145-151.

NaviA P., D., Villada C, H., \& Ayala A, A. (2016). Evaluaci;on mecánica de Bioplasticos semirrígidos elaborados con harina de yuca. Scielo, 5-9.

Navia-Porras, D., \& Bejarano-Arana, N. (2018). Evaluación de propiedades f;isicas de bioplásticos Termo-comprimidos elaborados con arina de yuca. Scielo, 4-9.

Salgado Garciglia, R. (2016). Bioplásticos: Productos Biodegradables. Saber Mas Revista de Divulgación, 1-3.

Sayavedra-Delgado, J., \& Rodríguez-Maecker, R. (2017). Desarrollo de bioplásticos a partir de tortas. Congreso de Ciencia y Tecnologia, 3-4. 
TUNJANO, V. (2016). Estudio de las de las propiedades térmicas y mecánicas del almidón termoplástico (TPS) reforzado con nanoarcilla. Revista latinoamericana de metalurgia y materiales, 29-36. 


\section{PARA CITAR EL ARTÍCULO INDEXADO.}

Barahona Alvear, N., Tixi Toapanta, H. P., Hugo Calderón, S., \& Barahona Alvear, L. (2019). Biopolímeros capaces de reemplazar a los plásticos tradicionales. Ciencia Digital, 3(4), 138-155. https://doi.org/10.33262/cienciadigital.v3i4.954

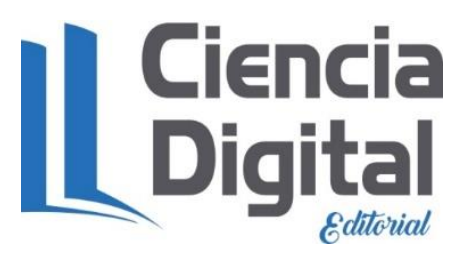

El artículo que se publica es de exclusiva responsabilidad de los autores y no necesariamente reflejan el pensamiento de la Revista Ciencia Digital.

El artículo queda en propiedad de la revista y, por tanto, su publicación parcial y/o total en otro medio tiene que ser autorizado por el director de la Revista Ciencia Digital.
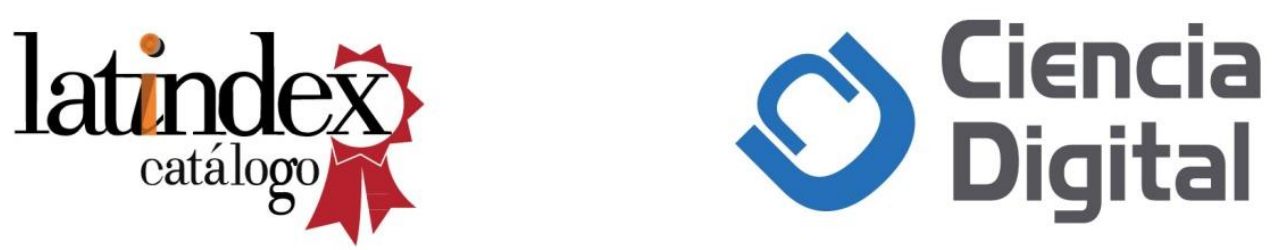